\title{
Effectiveness and Safety of Apixaban Versus Warfarin Among Older Patients with Venous Thromboembolism with Different Demographics and Socioeconomic Status
}

\author{
Alexander T. Cohen (D) · Janvi Sah · Amol D. Dhamane · \\ Theodore Lee · Lisa Rosenblatt · Patrick Hlavacek · Birol Emir · \\ Allison Keshishian · Huseyin Yuce · Xuemei Luo
}

Received: June 29, 2021 / Accepted: September 8, 2021 / Published online: September 27, 2021

(C) The Author(s) 2021

\section{ABSTRACT}

Introduction: Impact of demographics and socioeconomic status (SES) on anticoagulant treatment outcomes among patients with venous thromboembolism (VTE) is not well understood. This study evaluated risks of recurrent VTE, major bleeding (MB), and clinically relevant non-major bleeding (CRNMB) among older patients with VTE initiating

Supplementary Information The online version contains supplementary material available at https:// doi.org/10.1007/s12325-021-01918-0.

\footnotetext{
A. T. Cohen ( $\square)$

Department of Hematological Medicine, Guy's and St Thomas' Hospitals, Guy's and St Thomas' NHS Foundation Trust, King's College London, Westminster Bridge Road, London, UK e-mail: alexander.cohen@kcl.ac.uk

J. Sah · A. Keshishian

STATinMED Research, Ann Arbor, MI, USA

A. D. Dhamane - L. Rosenblatt

Bristol Myers Squibb Company, Lawrenceville, NJ, USA

T. Lee $\cdot$ P. Hlavacek · B. Emir

Pfizer, New York, NY, USA

H. Yuce

New York City College of Technology, City

University of New York, New York, NY, USA

X. Luo

Pfizer, Groton, CT, USA
}

apixaban or warfarin stratified by demographics and SES.

Methods: Adult patients ( $\geq 65$ years) who initiated apixaban or warfarin after a VTE event were selected from the US CMS Medicare database (September 2014-December 2017). Stabilized inverse probability treatment weighting (IPTW) was used to balance patient characteristics between treatment cohorts. Patients were stratified by age, gender, race, and SES. For each subgroup, Cox proportional hazard models were used to evaluate if there was a significant interaction $(p<0.10)$ between treatment and subgroup for recurrent VTE, MB, and CRNMB.

Results: In total, 22,135 apixaban and 45,840 warfarin patients with VTE were included. PostIPTW, patient characteristics were balanced between treatment cohorts. In older patients, apixaban treatment was associated with significantly lower risks of recurrent VTE (hazard ratio [HR] 0.64; 95\% confidence interval [CI] 0.52-0.79), MB (HR 0.65; 95\% CI 0.57-0.75), and CRNMB (HR 0.79; 95\% CI 0.75-0.85) versus warfarin. When stratified by demographics and SES, higher incidence rates of recurrent VTE, $\mathrm{MB}$, and CRNMB were observed for black vs white patients and patients with lower vs higher SES. Comparison of apixaban with warfarin by different demographic and SES subgroups showed generally consistent results as the overall analysis. For most subgroups, no significant interaction was observed between 
treatment and subgroup strata for recurrent VTE, MB, and CRNMB.

Conclusion: Among older patients with VTE initiating apixaban or warfarin, higher rates of recurrent VTE and bleeding were observed in black patients and patients with lower SES. Apixaban had a lower risk of recurrent VTE, MB, and CRNMB compared to warfarin. Analyses of demographic and SES subgroups showed consistent findings.

Keywords: Apixaban; Medicare; Race; Socioeconomic status; Venous thromboembolism

\section{Key Summary Points}

Why carry out this study?

Venous thromboembolism (VTE) is a common cause of preventable deaths in older hospitalized patients.

There has been a lack of evidence about potential impact of demographics and socioeconomic status on anticoagulant treatment outcomes in patients with VTE.

This study aimed to evaluate the risk of recurrent VTE, major bleeding (MB), and clinically relevant non-MB (CRNMB) among older patients with VTE initiating apixaban or warfarin stratified by demographics and socioeconomic status in routine clinical practice.

\section{What was learned from this study?}

Among older patients with VTE, black patients and those with lower socioeconomic status tended to have higher incidence rates of recurrent VTE, $\mathrm{MB}$, and CRNMB compared to their counterparts.

When compared to warfarin, apixaban had a lower risk of recurrent VTE, MB, and CRNMB among patients with VTE. Analyses of the demographics and socioeconomic subgroups showed generally consistent findings.

\section{INTRODUCTION}

Venous thromboembolism (VTE) is a common cause of preventable death especially in older hospitalized patients $[1,2]$. Acquired risk factors for VTE include age, obesity, smoking, surgery, immobility, hospitalization, pregnancy, hormone therapy, malignancy, and other comorbid medical conditions [3]. There exist racial differences in the incidence of VTE-black patients have a significantly higher rate of VTE and a higher rate of complications such as death, major bleeding (MB), and recurrent VTE compared to white patients [4-6]. Another study showed that black Americans had a $30-60 \%$ higher incidence rate of VTE than white Americans despite having lower rates of the two genetic mutations known to increase the risk of VTE [7]. While VTE can occur in all races, gender, and age groups, there is a significantly higher prevalence of VTE among black and older patients $[8,9]$.

Clinical guidelines recommend the use of direct oral anticoagulants (DOACs; including apixaban, dabigatran, edoxaban, rivaroxaban) and warfarin for the treatment of VTE $[10,11]$. However, the effects of demographics and socioeconomic status (SES) on DOAC treatment outcomes are not well understood. Some studies have found associations between SES and the use of DOACs vs vitamin $\mathrm{K}$ antagonist in patients with VTE-patients with a lower household income were less likely to use DOACs compared to those with a higher household income [12]. The difference in DOAC treatment rate by SES may lead to disparity in health outcomes such as the risk of developing recurrent VTE. Additionally, patients may respond to anticoagulant treatments differently because of different age, gender, race, and SES.

However, few clinical trials and real-world studies have evaluated the impact of demographics and SES on anticoagulant treatment outcomes among patients with VTE. Minorities and patients with lower SES have been underrepresented in clinical trials [13], and evidence on the effectiveness and safety of anticoagulants in patients with VTE with different 
demographics and SES is sparse. Therefore, this study aimed to evaluate the risk of recurrent VTE, MB, and clinically relevant non-major bleeding (CRNMB) among older patients with VTE initiating apixaban or warfarin stratified by demographics and SES in routine clinical practice.

\section{METHODS}

\section{Data Source and Patient Selection}

This retrospective analysis utilized data from the United States (US) Centers for Medicare and Medicaid Services (CMS) fee-for-service Medicare database. Patients with a VTE diagnosis (based on International Classification of Diseases, 9th and 10th Revision codes) in any position in the inpatient or outpatient setting were identified between September 1, 2014 and December 31, 2017. Patients were required to have at least one pharmacy claim for apixaban or warfarin during the 30-day period following the index VTE event. The first warfarin or apixaban prescription claim was designated as the index date. Patients aged at least 65 years on the index date with continuous health plan enrollment, including medical and pharmacy benefits, for at least 6 months before the index VTE event until the index date were included in the study population. Additional selection criteria are listed in Fig. 1.

The baseline period was defined as 6 months prior to and including the index date; the follow-up period included the day after the index date through the earliest of index therapy discontinuation, switch to another oral anticoagulant or parenteral anticoagulant, health plan disenrollment, death, the end of the study period, or 6-months post-index date. This study employed an on-treatment approach and therefore did not consider events that occurred after a patient switched or discontinued index treatment.

\section{Study Variables}

Recurrent VTE and MB were defined by primary diagnosis in the inpatient setting [14-18]. CRNMB was identified by either an inpatient admission with a secondary diagnosis code for non-critical sites of bleeding (excluded if $\mathrm{MB}$ occurred before the CRNM bleed or during the same hospitalization) or by a diagnosis code for gastrointestinal bleeding or other selected noncritical site of bleeding in the outpatient setting. This approach aligns with the current recommendation of the International Society on Thrombosis and Haemostasis [14-19].

Demographic variables including age, gender, and race were measured on the index date. Age was categorized as 65-79 years and $\geq 80$ years. Gender was categorized as male and female. Race was categorized as white, black, and other race (including Asian, Hispanics, North American native, other, and unknown races). Two SES factors were considered-one was Medicare Medicaid dual eligibility or Part D low income subsidy (LIS) anytime during the baseline period. Another was SES status constructed for each US zip code using data on income, education, and occupation from the 2015 US census. This information was then linked to the patient's zip code of residence on the index date to define the SES for the patient [20, 21]. Clinical characteristics (such as the Deyo-Charlson comorbidity index, baseline comorbidities, falls, fracture/trauma involving lower extremities, selected surgeries, and baseline medications) were evaluated during the baseline period. VTE-related variables such as type of index VTE event and index VTE diagnosis were measured during the index VTE event date.

\section{Statistical Methods}

Patient characteristics were balanced between the treatment cohorts using stabilized inverse probability treatment weighting (IPTW); baseline demographics, clinical characteristics, and VTE-related variables were covariates included in the model. IPTW weights were stabilized by multiplying the original weights with a 
Patients with a VTE diagnosis in the inpatient or outpatient setting between September 1, 2014 to December 31, 2017. The first VTE diagnosis was considered as the 'index VTE event'.

Remaining $\mathrm{N}=1,453,486$

Patients of age $\geq 65$ years on the index date for Medicare database who had $\geq 1$ pharmacy claim for apixaban or warfarin during the 30 day identification period following the index VTE event. The first warfarin or apixaban prescription was designated as the 'index date'.

Remaining $\mathrm{N}=\mathbf{2 5 0 , 1 2 3}$

Patients with continuous health plan enrollment with medical and pharmacy benefits for at least 6 months before the index VTE event
until the index date
Remaining $\mathbf{N = 2 3 1 , 3 3 7}$

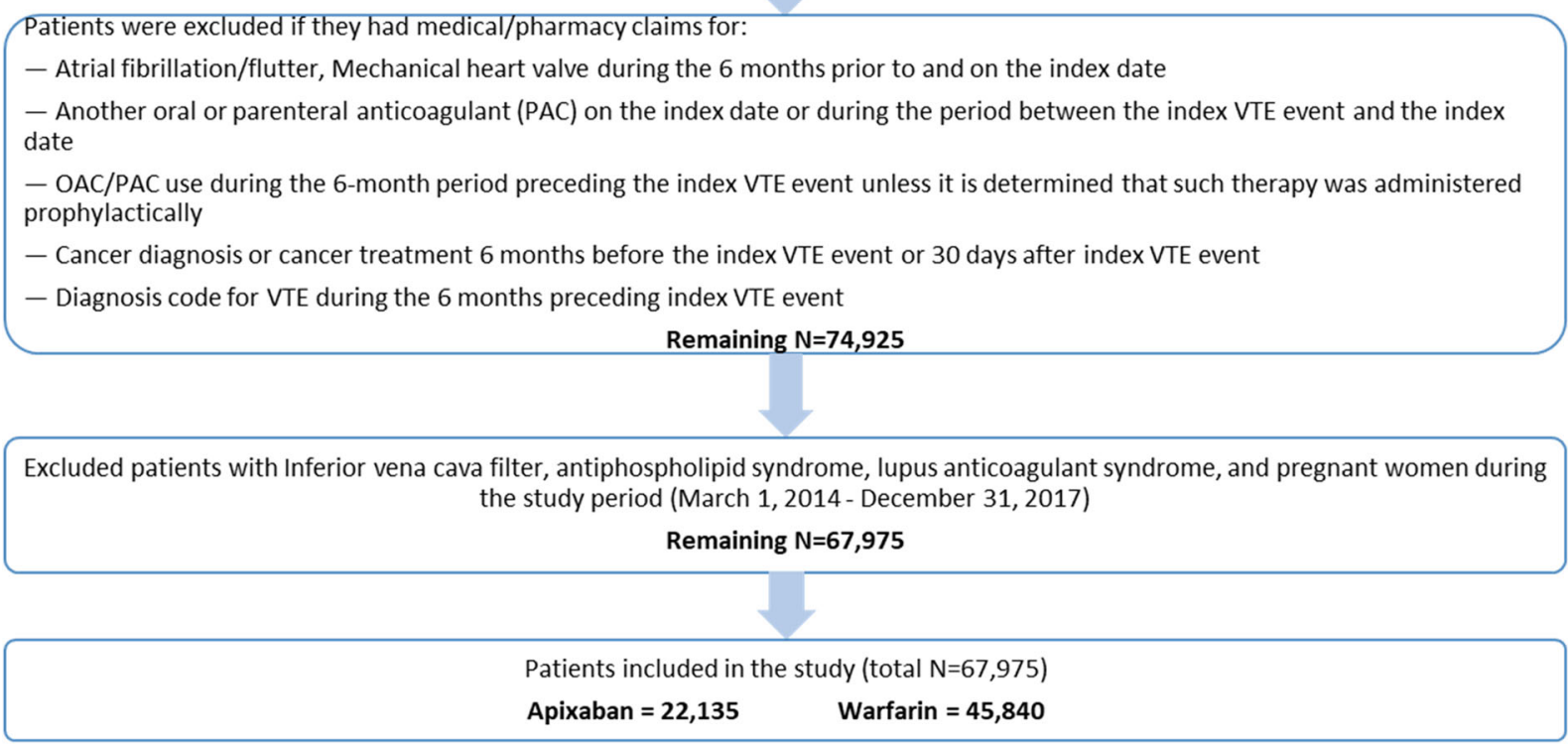

Fig. 1 Patient selection criteria

constant that was equal to the expected value of being in the treatment or comparison cohorts [22-24]. The risk of recurrent VTE, MB, and CRNMB was evaluated by using Cox proportional hazard models. Interaction analyses were conducted using Cox models to evaluate whether treatment effects differ by age (65-79 vs $\geq 80$ years), gender (male vs female), race (white vs black), SES (low vs medium vs high), and dual Medicare/Medicaid eligibility or Part-D LIS (yes vs no). For each of the demographic and SES subgroups, a statistical significance $(p<0.10)$ of the interaction between the treatment and subgroup strata on effectiveness and safety was evaluated.

\section{Compliance with Ethics Guidelines}

This retrospective database analysis did not involve the collection, use, or transmittal of individual identifiable data. As such, institutional review board (IRB) approval to conduct this study was not required and considered exempt according to 45CFR46.101(b)(4): existing data and specimens-no identifiers. Both the data set itself and the security of the offices where the data are housed meet the requirements of the Health Insurance Portability and Accountability Act (HIPAA) of 1996. 


\section{RESULTS}

During the study period, approximately 1.4 million patients had a VTE event in the Medicare database; after application of the selection criteria, 22,135 (32.6\%) patients who initiated apixaban and 45,840 (67.4\%) patients who initiated warfarin were included in the study (Fig. 1). Table 1 shows demographics, SES, and clinical characteristics for the apixaban and warfarin cohorts before and after IPTW. After IPTW, all patient characteristics were well balanced (Table 1). For both treatment cohorts, most patients were aged 65-79 years (apixaban, $62.6 \%$; warfarin, $62.6 \%$ ), followed by $\geq 80$ years (apixaban, 37.4\%; warfarin, 37.4\%). The majority of patients were female (apixaban, 62.9\%; warfarin, 62.9\%) and white (apixaban, 84.2\%; warfarin, $83.7 \%$ ) across the two cohorts. The largest proportion of patients had a high SES (apixaban, 45.2\%; warfarin, 42.2\%), followed by medium (apixaban, 30.2\%; warfarin, $32.6 \%$ ), and low (apixaban, 22.7\%; warfarin, $23.4 \%)$. One-third of patients also had Medicare/Medicaid dual-eligibility and/or Part D low income subsidy (apixaban, 28.5\%; warfarin, $30.5 \%$ ) during the baseline period. During the follow-up period, apixaban patients had significantly lower risk of recurrent VTE (hazard ratio [HR] 0.64; 95\% confidence interval [CI] 0.52-0.79), MB (HR 0.65; 95\% CI 0.57-0.75), and CRNMB (HR 0.79; 95\% CI $0.75-0.85$ ) compared to warfarin patients.

The baseline characteristics for older patients with VTE on apixaban vs warfarin stratified by age, gender, race, SES status, and dual eligibility/Part-D LIS are listed in Supplemental Material. For both apixaban and warfarin cohorts, higher mean comorbidity index was observed in patients aged $\geq 80$ vs 65-79 years (apixaban, 3.2 vs 2.7; warfarin, 3.2 vs 2.7 ), low vs high SES (apixaban, 3.5 vs 2.6; warfarin, 3.3 vs 2.7 ), and dual eligibility/Part-D LIS yes vs no (apixaban, 3.9 vs 2.5 ; warfarin, 3.8 vs 2.5 ) and black vs white patients (apixaban, 4.1 vs 2.7 ; warfarin, 4.1 vs 2.7 ). Additionally, during the follow-up, the incidence rates per 100 person-years for recurrent VTE were numerically higher for black vs white patients (apixaban, 2.0 vs 1.4; warfarin,
3.3 vs 2.2 ) and patients with low vs high SES (apixaban, 2.6 vs 1.3; warfarin, 3.2 vs 2.0 ) and dual eligibility/Part-D LIS yes vs no (apixaban, 2.2 vs 1.3; warfarin, 3.1 vs 2.0) (Fig. 2). Similarly, the incidence rates per 100 person-years for MB were numerically higher for patients aged $\geq 80$ vs $65-79$ years (apixaban, 5.4 vs 3.1; warfarin, 7.4 vs 5.0 ), female vs male (apixaban, 4.5 vs 3.1 ; warfarin, 6.4 vs 5.0 ), black vs white patients (apixaban, 7.4 vs 3.5; warfarin 10.1 vs 5.3 ), low vs high SES (apixaban, 5.7 vs 3.2; warfarin, 7.0 vs 5.1), and dual eligibility/Part-D LIS yes vs no (apixaban, 6.3 vs 3.1; warfarin, 8.6 vs 4.8 ) (Fig. 3).

Figures 2, 3, and 4 show the data of apixaban vs warfarin on risks of recurrent VTE, MB, and CRNMB stratified by demographic and SES factors. No significant interaction was observed between the treatment and the following subgroups on recurrent VTE (Fig. 2) and MB (Fig. 3): age, sex, race, SES, and dual eligibility/LIS. Across all subgroup strata, apixaban patients had a lower incidence rate of recurrent VTE and MB compared to warfarin patients. For CRNMB, no significant interaction was observed between the treatment and the following subgroups: sex, race, and dual eligibility/LIS (Fig. 4). However, there were significant interactions between the treatment and age (interaction $p$ value $=0.001$ ) and between the treatment and SES (interaction $p$ value $=0.012$ ) on CRNMB. Apixaban trended towards a lower risk of CRNMB across both age groups, but the treatment effect on CRNMB was larger for patients aged 65-79 years. Similarly, apixaban trended towards a lower risk of CRNMB across all three SES strata, but the treatment effect was larger for those with medium and high SES (Fig. 4).

\section{DISCUSSION}

There has been a lack of evidence about the potential impact of demographic and SES factors on anticoagulant treatment outcomes in patients with VTE. To help fill in some of the evidence gaps, this study compared the risk of recurrent VTE, MB, and CRNMB among older patients with VTE who initiated apixaban vs warfarin stratified by demographic and SES 
Table 1 Baseline and clinical characteristics among older patients with VTE that initiated apixaban vs warfarin pre and post IPTW

\begin{tabular}{|c|c|c|c|c|c|c|}
\hline & \multicolumn{3}{|c|}{ Pre IPTW } & \multicolumn{3}{|c|}{ Post IPTW** } \\
\hline & $\begin{array}{l}\text { Warfarin } \\
\text { cohort }\end{array}$ & $\begin{array}{l}\text { Apixaban } \\
\text { cohort }\end{array}$ & STD $^{*}$ & $\begin{array}{l}\text { Warfarin } \\
\text { cohort }\end{array}$ & $\begin{array}{l}\text { Apixaban } \\
\text { cohort }\end{array}$ & STD $^{*}$ \\
\hline Sample size & 45,840 & 22,135 & & 45,840 & 22,135 & \\
\hline Age (in years), mean (SD) & $77.2(8.1)$ & $77.7(8.2)$ & 6.01 & $77.3(8.1)$ & $77.4(8.2)$ & 1.57 \\
\hline \multicolumn{7}{|l|}{ Age category (in years), $n(\%)$} \\
\hline $65-79$ & $\begin{array}{l}29,021 \\
\quad(63.3 \%)\end{array}$ & $\begin{array}{l}13,548 \\
\quad(61.2 \%)\end{array}$ & 4.34 & $\begin{array}{l}28,703 \\
\quad(62.6 \%)\end{array}$ & $\begin{array}{l}13,848 \\
\quad(62.6 \%)\end{array}$ & 0.11 \\
\hline$\geq 80$ & $\begin{array}{l}16,819 \\
\quad(36.7 \%)\end{array}$ & $\begin{array}{l}8587 \\
\quad(38.8 \%)\end{array}$ & 4.34 & $\begin{array}{l}17,137 \\
\quad(37.4 \%)\end{array}$ & $\begin{array}{l}8287 \\
\quad(37.4 \%)\end{array}$ & 0.11 \\
\hline \multicolumn{7}{|l|}{ Sex, $n(\%)$} \\
\hline Male & $\begin{array}{l}16,959 \\
\quad(37.0 \%)\end{array}$ & $\begin{array}{l}8300 \\
\quad(37.5 \%)\end{array}$ & 1.04 & $\begin{array}{l}17,019 \\
\quad(37.1 \%)\end{array}$ & $\begin{array}{l}8202 \\
\quad(37.1 \%)\end{array}$ & 0.15 \\
\hline Female & $\begin{array}{l}28,881 \\
\quad(63.0 \%)\end{array}$ & $\begin{array}{l}13,835 \\
\quad(62.5 \%)\end{array}$ & 1.04 & $\begin{array}{l}28,821 \\
\quad(62.9 \%)\end{array}$ & $\begin{array}{l}13,933 \\
\quad(62.9 \%)\end{array}$ & 0.15 \\
\hline \multicolumn{7}{|l|}{ Race, $n(\%)$} \\
\hline White & $\begin{array}{l}38,487 \\
\quad(84.0 \%)\end{array}$ & $\begin{array}{l}18,575 \\
(83.9 \%)\end{array}$ & 0.12 & $\begin{array}{l}38,371 \\
\quad(83.7 \%)\end{array}$ & $\begin{array}{l}18,636 \\
\quad(84.2 \%)\end{array}$ & 1.33 \\
\hline African American & $\begin{array}{l}5239 \\
\quad(11.4 \%)\end{array}$ & $\begin{array}{l}2535 \\
\quad(11.5 \%)\end{array}$ & 0.07 & $\begin{array}{l}5404 \\
\quad(11.8 \%)\end{array}$ & $\begin{array}{l}2428 \\
\quad(11.0 \%)\end{array}$ & 2.58 \\
\hline Other categories & $\begin{array}{l}2114 \\
\quad(4.6 \%)\end{array}$ & $\begin{array}{l}1025 \\
(4.6 \%)\end{array}$ & 0.09 & $\begin{array}{l}2065 \\
\quad(4.5 \%)\end{array}$ & $\begin{array}{l}1070 \\
\quad(4.8 \%)\end{array}$ & 1.66 \\
\hline \multicolumn{7}{|l|}{ SES status, $n(\%)$} \\
\hline Low & $\begin{array}{l}10,374 \\
\quad(22.6 \%)\end{array}$ & $\begin{array}{l}5320 \\
\quad(24.0 \%)\end{array}$ & 3.32 & $\begin{array}{l}10,733 \\
\quad(23.4 \%)\end{array}$ & $\begin{array}{l}5029 \\
\quad(22.7 \%)\end{array}$ & 1.65 \\
\hline Medium & $\begin{array}{l}15,083 \\
\quad(32.9 \%)\end{array}$ & $\begin{array}{l}6600 \\
\quad(29.8 \%)\end{array}$ & 6.66 & $\begin{array}{l}14,959 \\
(32.6 \%)\end{array}$ & $\begin{array}{l}6684 \\
\quad(30.2 \%)\end{array}$ & 5.25 \\
\hline High & $\begin{array}{l}19,567 \\
\quad(42.7 \%)\end{array}$ & $\begin{array}{l}9792 \\
\quad(44.2 \%)\end{array}$ & 3.13 & $\begin{array}{l}19,324 \\
\quad(42.2 \%)\end{array}$ & $\begin{array}{l}10,008 \\
\quad(45.2 \%)\end{array}$ & 6.17 \\
\hline Missing & $\begin{array}{l}816 \\
\quad(1.8 \%)\end{array}$ & $\begin{array}{l}423 \\
\quad(1.9 \%)\end{array}$ & 0.97 & $\begin{array}{l}824 \\
\quad(1.8 \%)\end{array}$ & $\begin{array}{l}414 \\
\quad(1.9 \%)\end{array}$ & 0.52 \\
\hline $\begin{array}{l}\text { Medicare/Medicaid dual-eligibility and/or Part } \mathrm{D} \text { low } \\
\text { income subsidy during the baseline period, } n(\%)\end{array}$ & $\begin{array}{l}13,857 \\
(30.2 \%)\end{array}$ & $\begin{array}{l}6385 \\
(28.8 \%)\end{array}$ & 3.03 & $\begin{array}{l}13,990 \\
(30.5 \%)\end{array}$ & $\begin{array}{l}6319 \\
(28.5 \%)\end{array}$ & 4.32 \\
\hline Type of index VTE event, $n$ (\%) & & & & & & \\
\hline
\end{tabular}


Table 1 continued

\begin{tabular}{|c|c|c|c|c|c|c|}
\hline & Pre IPTW & & & Post IPT & & \\
\hline & $\begin{array}{l}\text { Warfarin } \\
\text { cohort }\end{array}$ & $\begin{array}{l}\text { Apixaban } \\
\text { cohort }\end{array}$ & STD $^{*}$ & $\begin{array}{l}\text { Warfarin } \\
\text { cohort }\end{array}$ & $\begin{array}{l}\text { Apixaban } \\
\text { cohort }\end{array}$ & STD* $^{*}$ \\
\hline Inpatient & $\begin{array}{l}29,977 \\
\quad(65.4 \%)\end{array}$ & $\begin{array}{l}14,391 \\
\quad(65.0 \%)\end{array}$ & 0.80 & $\begin{array}{l}29,907 \\
\quad(65.2 \%)\end{array}$ & $\begin{array}{l}14,418 \\
\quad(65.1 \%)\end{array}$ & 0.22 \\
\hline Outpatient & $\begin{array}{l}15,863 \\
(34.6 \%)\end{array}$ & $\begin{array}{l}7744 \\
\quad(35.0 \%)\end{array}$ & 0.80 & $\begin{array}{l}15,933 \\
\quad(34.8 \%)\end{array}$ & $\begin{array}{l}7717 \\
\quad(34.9 \%)\end{array}$ & 0.22 \\
\hline Index VTE diagnosis, $n(\%)$ & & & & & & \\
\hline DVT only & $\begin{array}{l}24,772 \\
\quad(54.0 \%)\end{array}$ & $\begin{array}{l}11,669 \\
\quad(52.7 \%)\end{array}$ & 2.65 & $\begin{array}{l}24,601 \\
\quad(53.7 \%)\end{array}$ & $\begin{array}{l}11,913 \\
\quad(53.8 \%)\end{array}$ & 0.31 \\
\hline PE with DVT & $\begin{array}{l}7091 \\
\quad(15.5 \%)\end{array}$ & $\begin{array}{l}3502 \\
\quad(15.8 \%)\end{array}$ & 0.97 & $\begin{array}{l}7145 \\
\quad(15.6 \%)\end{array}$ & $\begin{array}{l}3448 \\
\quad(15.6 \%)\end{array}$ & 0.02 \\
\hline PE without DVT & $\begin{array}{l}13,977 \\
\quad(30.5 \%)\end{array}$ & $\begin{array}{l}6964 \\
\quad(31.5 \%)\end{array}$ & 2.10 & $\begin{array}{l}14,094 \\
\quad(30.7 \%)\end{array}$ & $\begin{array}{l}6773 \\
\quad(30.6 \%)\end{array}$ & 0.32 \\
\hline Deyo-Charlson comorbidity index, mean (SD) & $2.9(2.5)$ & $2.9(2.5)$ & 1.40 & $2.9(2.5)$ & $2.9(2.5)$ & 0.37 \\
\hline Baseline comorbidity, $n(\%)$ & & & & & & \\
\hline AIDS & $\begin{array}{l}128 \\
\quad(0.3 \%)\end{array}$ & $46(0.2 \%)$ & 1.45 & $\begin{array}{l}130 \\
\quad(0.3 \%)\end{array}$ & $48(0.2 \%)$ & 1.29 \\
\hline Alcohol abuse & $\begin{array}{l}1178 \\
(2.6 \%)\end{array}$ & $\begin{array}{l}525 \\
\quad(2.4 \%)\end{array}$ & 1.28 & $\begin{array}{l}1152 \\
(2.5 \%)\end{array}$ & $\begin{array}{l}561 \\
(2.5 \%)\end{array}$ & 0.13 \\
\hline Anemia & $\begin{array}{l}17,520 \\
\quad(38.2 \%)\end{array}$ & $\begin{array}{l}8026 \\
\quad(36.3 \%)\end{array}$ & 4.06 & $\begin{array}{l}17,246 \\
\quad(37.6 \%)\end{array}$ & $\begin{array}{l}8340 \\
\quad(37.7 \%)\end{array}$ & 0.11 \\
\hline Central venous catheter & $\begin{array}{l}4047 \\
(8.8 \%)\end{array}$ & $\begin{array}{l}1597 \\
\quad(7.2 \%)\end{array}$ & 5.94 & $\begin{array}{l}3810 \\
\quad(8.3 \%)\end{array}$ & $\begin{array}{l}1835 \\
(8.3 \%)\end{array}$ & 0.08 \\
\hline Cerebrovascular disease & $\begin{array}{l}7918 \\
\quad(17.3 \%)\end{array}$ & $\begin{array}{l}3786 \\
\quad(17.1 \%)\end{array}$ & 0.45 & $\begin{array}{l}8006 \\
\quad(17.5 \%)\end{array}$ & $\begin{array}{l}3724 \\
\quad(16.8 \%)\end{array}$ & 1.70 \\
\hline Coagulation defects & $\begin{array}{l}4429 \\
\quad(9.7 \%)\end{array}$ & $\begin{array}{l}1870 \\
\quad(8.4 \%)\end{array}$ & 4.23 & $\begin{array}{l}4254 \\
\quad(9.3 \%)\end{array}$ & $\begin{array}{l}2064 \\
\quad(9.3 \%)\end{array}$ & 0.15 \\
\hline Ischemic heart/coronary artery disease & $\begin{array}{l}15,724 \\
(34.3 \%)\end{array}$ & $\begin{array}{l}8111 \\
\quad(36.6 \%)\end{array}$ & 4.90 & $\begin{array}{l}16,071 \\
\quad(35.1 \%)\end{array}$ & $\begin{array}{l}7757 \\
\quad(35.0 \%)\end{array}$ & 0.03 \\
\hline Dementia & $\begin{array}{l}4868 \\
\quad(10.6 \%)\end{array}$ & $\begin{array}{l}3041 \\
\quad(13.7 \%)\end{array}$ & 9.55 & $\begin{array}{l}4934 \\
\quad(10.8 \%)\end{array}$ & $\begin{array}{l}2996 \\
\quad(13.5 \%)\end{array}$ & 8.48 \\
\hline Dyspepsia or stomach discomfort & $\begin{array}{l}11,168 \\
\quad(24.4 \%)\end{array}$ & $\begin{array}{l}5336 \\
\quad(24.1 \%)\end{array}$ & 0.60 & $\begin{array}{l}11,150 \\
\quad(24.3 \%)\end{array}$ & $\begin{array}{l}5413 \\
\quad(24.5 \%)\end{array}$ & 0.31 \\
\hline Hemiplegia or paraplegia & $\begin{array}{l}1157 \\
(2.5 \%)\end{array}$ & $\begin{array}{l}542 \\
\quad(2.4 \%)\end{array}$ & 0.48 & $\begin{array}{l}1157 \\
(2.5 \%)\end{array}$ & $\begin{array}{l}562 \\
\quad(2.5 \%)\end{array}$ & 0.11 \\
\hline
\end{tabular}


Table 1 continued

\begin{tabular}{|c|c|c|c|c|c|c|}
\hline & \multicolumn{3}{|c|}{ Pre IPTW } & \multicolumn{3}{|c|}{ Post IPTW** } \\
\hline & $\begin{array}{l}\text { Warfarin } \\
\text { cohort }\end{array}$ & $\begin{array}{l}\text { Apixaban } \\
\text { cohort }\end{array}$ & STD $^{*}$ & $\begin{array}{l}\text { Warfarin } \\
\text { cohort }\end{array}$ & $\begin{array}{l}\text { Apixaban } \\
\text { cohort }\end{array}$ & STD $^{*}$ \\
\hline Hyperlipidemia & $\begin{array}{l}27,678 \\
\quad(60.4 \%)\end{array}$ & $\begin{array}{l}14,048 \\
(63.5 \%)\end{array}$ & 6.36 & $\begin{array}{l}28,129 \\
\quad(61.4 \%)\end{array}$ & $\begin{array}{l}13,563 \\
(61.3 \%)\end{array}$ & 0.19 \\
\hline Obesity & $\begin{array}{l}11,558 \\
(25.2 \%)\end{array}$ & $\begin{array}{l}5794 \\
\quad(26.2 \%)\end{array}$ & 2.20 & $\begin{array}{l}11,689 \\
(25.5 \%)\end{array}$ & $\begin{array}{l}5631 \\
\quad(25.4 \%)\end{array}$ & 0.14 \\
\hline Pneumonia & $\begin{array}{l}7893 \\
\quad(17.2 \%)\end{array}$ & $\begin{array}{l}3912 \\
\quad(17.7 \%)\end{array}$ & 1.20 & $\begin{array}{l}7980 \\
(17.4 \%)\end{array}$ & $\begin{array}{l}3868 \\
\quad(17.5 \%)\end{array}$ & 0.17 \\
\hline Rheumatologic disease & $\begin{array}{l}3060 \\
\quad(6.7 \%)\end{array}$ & $\begin{array}{l}1455 \\
(6.6 \%)\end{array}$ & 0.41 & $\begin{array}{l}3044 \\
(6.6 \%)\end{array}$ & $\begin{array}{l}1465 \\
(6.6 \%)\end{array}$ & 0.08 \\
\hline Sleep apnea & $\begin{array}{l}6394 \\
\quad(13.9 \%)\end{array}$ & $\begin{array}{l}3086 \\
\quad(13.9 \%)\end{array}$ & 0.02 & $\begin{array}{l}6375 \\
(13.9 \%)\end{array}$ & $\begin{array}{l}3054 \\
\quad(13.8 \%)\end{array}$ & 0.32 \\
\hline Spinal cord injury & $\begin{array}{l}130 \\
(0.3 \%)\end{array}$ & $46(0.2 \%)$ & 1.53 & $\begin{array}{l}119 \\
(0.3 \%)\end{array}$ & $59(0.3 \%)$ & 0.10 \\
\hline Thrombophilia & $\begin{array}{l}1450 \\
(3.2 \%)\end{array}$ & $\begin{array}{l}627 \\
(2.8 \%)\end{array}$ & 1.94 & $\begin{array}{l}1397 \\
(3.0 \%)\end{array}$ & $\begin{array}{l}670 \\
(3.0 \%)\end{array}$ & 0.11 \\
\hline Varicose veins & $\begin{array}{l}1999 \\
\quad(4.4 \%)\end{array}$ & $\begin{array}{l}1059 \\
(4.8 \%)\end{array}$ & 2.03 & $\begin{array}{l}2065 \\
\quad(4.5 \%)\end{array}$ & $\begin{array}{l}996 \\
(4.5 \%)\end{array}$ & 0.02 \\
\hline Congestive heart failure & $\begin{array}{l}10,648 \\
\quad(23.2 \%)\end{array}$ & $\begin{array}{l}5233 \\
(23.6 \%)\end{array}$ & 0.97 & $\begin{array}{l}10,731 \\
(23.4 \%)\end{array}$ & $\begin{array}{l}5221 \\
\quad(23.6 \%)\end{array}$ & 0.42 \\
\hline Diabetes & $\begin{array}{l}17,825 \\
\quad(38.9 \%)\end{array}$ & $\begin{array}{l}8031 \\
\quad(36.3 \%)\end{array}$ & 5.38 & $\begin{array}{l}17,978 \\
\quad(39.2 \%)\end{array}$ & $\begin{array}{l}7900 \\
\quad(35.7 \%)\end{array}$ & 7.29 \\
\hline Hypertension & $\begin{array}{l}38,312 \\
\quad(83.6 \%)\end{array}$ & $\begin{array}{l}18,691 \\
\quad(84.4 \%)\end{array}$ & 2.36 & $\begin{array}{l}38,446 \\
\quad(83.9 \%)\end{array}$ & $\begin{array}{l}18,571 \\
\quad(83.9 \%)\end{array}$ & 0.08 \\
\hline Non-ESRD renal disease & $\begin{array}{l}9408 \\
\quad(20.5 \%)\end{array}$ & $\begin{array}{l}4615 \\
\quad(20.8 \%)\end{array}$ & 0.80 & $\begin{array}{l}9466 \\
(20.6 \%)\end{array}$ & $\begin{array}{l}4585 \\
(20.7 \%)\end{array}$ & 0.16 \\
\hline End stage renal disease & $\begin{array}{l}1588 \\
\quad(3.5 \%)\end{array}$ & $\begin{array}{l}449 \\
(2.0 \%)\end{array}$ & 8.79 & $\begin{array}{l}1375 \\
(3.0 \%)\end{array}$ & $\begin{array}{l}675 \\
\quad(3.0 \%)\end{array}$ & 0.28 \\
\hline Chronic liver disease & $\begin{array}{l}2861 \\
\quad(6.2 \%)\end{array}$ & $\begin{array}{l}1570 \\
\quad(7.1 \%)\end{array}$ & 3.41 & $\begin{array}{l}2807 \\
\quad(6.1 \%)\end{array}$ & $\begin{array}{l}1610 \\
(7.3 \%)\end{array}$ & 4.59 \\
\hline COPD & $\begin{array}{l}11,754 \\
\quad(25.6 \%)\end{array}$ & $\begin{array}{l}5814 \\
\quad(26.3 \%)\end{array}$ & 1.43 & $\begin{array}{l}11,858 \\
(25.9 \%)\end{array}$ & $\begin{array}{l}5800 \\
\quad(26.2 \%)\end{array}$ & 0.76 \\
\hline Peptic ulcer disease & $\begin{array}{l}1296 \\
(2.8 \%)\end{array}$ & $\begin{array}{l}533 \\
(2.4 \%)\end{array}$ & 2.63 & $\begin{array}{l}1267 \\
(2.8 \%)\end{array}$ & $\begin{array}{l}564 \\
(2.5 \%)\end{array}$ & 1.35 \\
\hline Inflammatory bowel disease & $\begin{array}{l}853 \\
\quad(1.9 \%)\end{array}$ & $\begin{array}{l}378 \\
(1.7 \%)\end{array}$ & 1.16 & $\begin{array}{l}829 \\
(1.8 \%)\end{array}$ & $\begin{array}{l}395 \\
\quad(1.8 \%)\end{array}$ & 0.19 \\
\hline
\end{tabular}


Table 1 continued

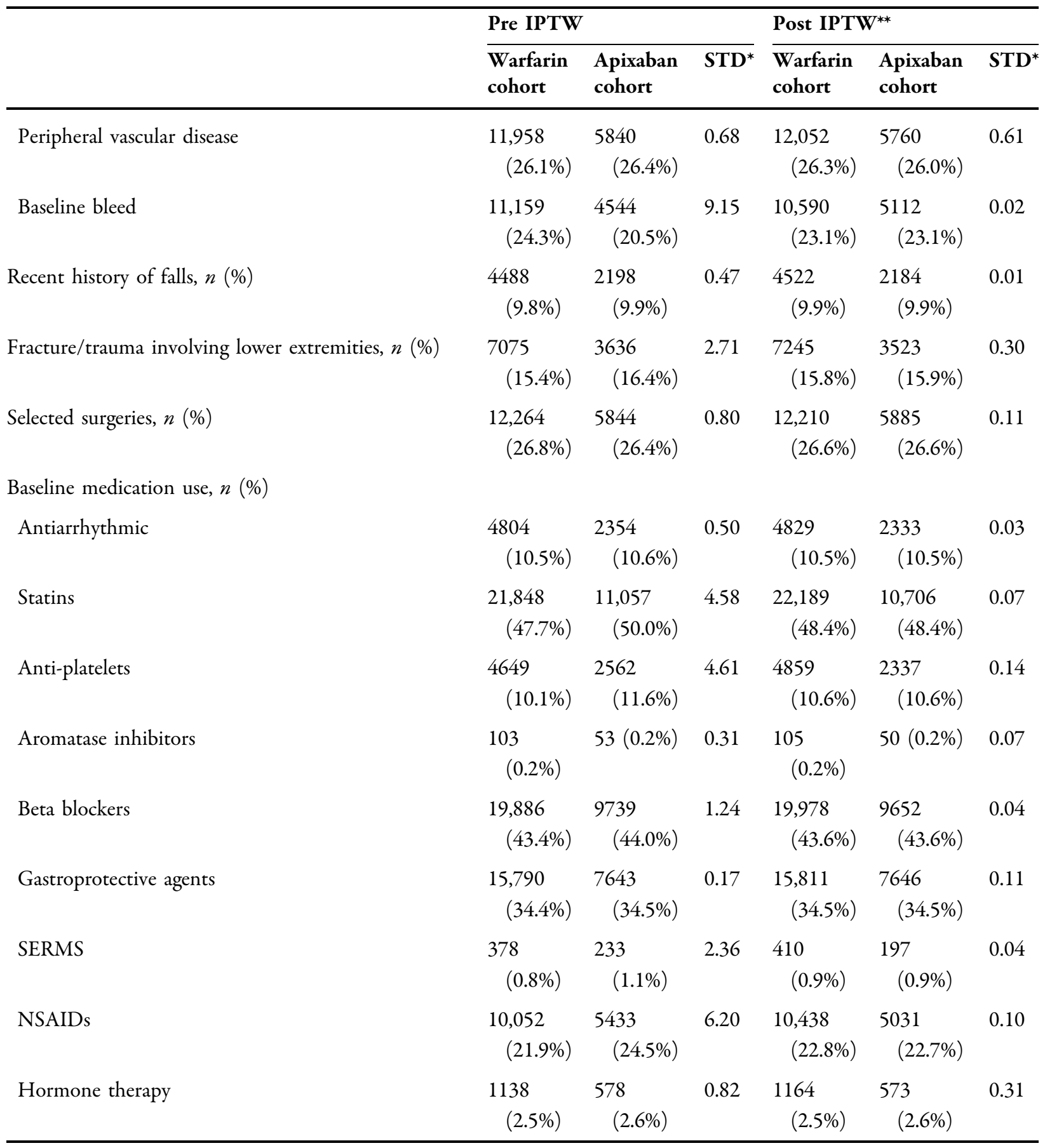

$A I D S$ acquired immunodeficiency syndrome, COPD chronic obstructive pulmonary disorder, $D V T$ deep vein thrombosis, ESRD end stage renal disease, IPTW inverse probability of treatment weighting, NSAIDs nonsteroidal anti-inflammatory drugs, $P E$ pulmonary embolism, SERMS selective estrogen receptor modulators, $S E S$ socioeconomic status, $S D$ standard deviation, $S T D$ standardized differences, VTE venous thromboembolism

${ }^{*}$ STD $=100 \times \mid$ actual standardized difference|. Standardized difference (STD) greater than 10 was considered significant ${ }^{* *}$ After weightings were applied, the values for the baseline variables were not whole numbers; therefore, as a result of rounding the sum of patients may not equal $100 \%$ 


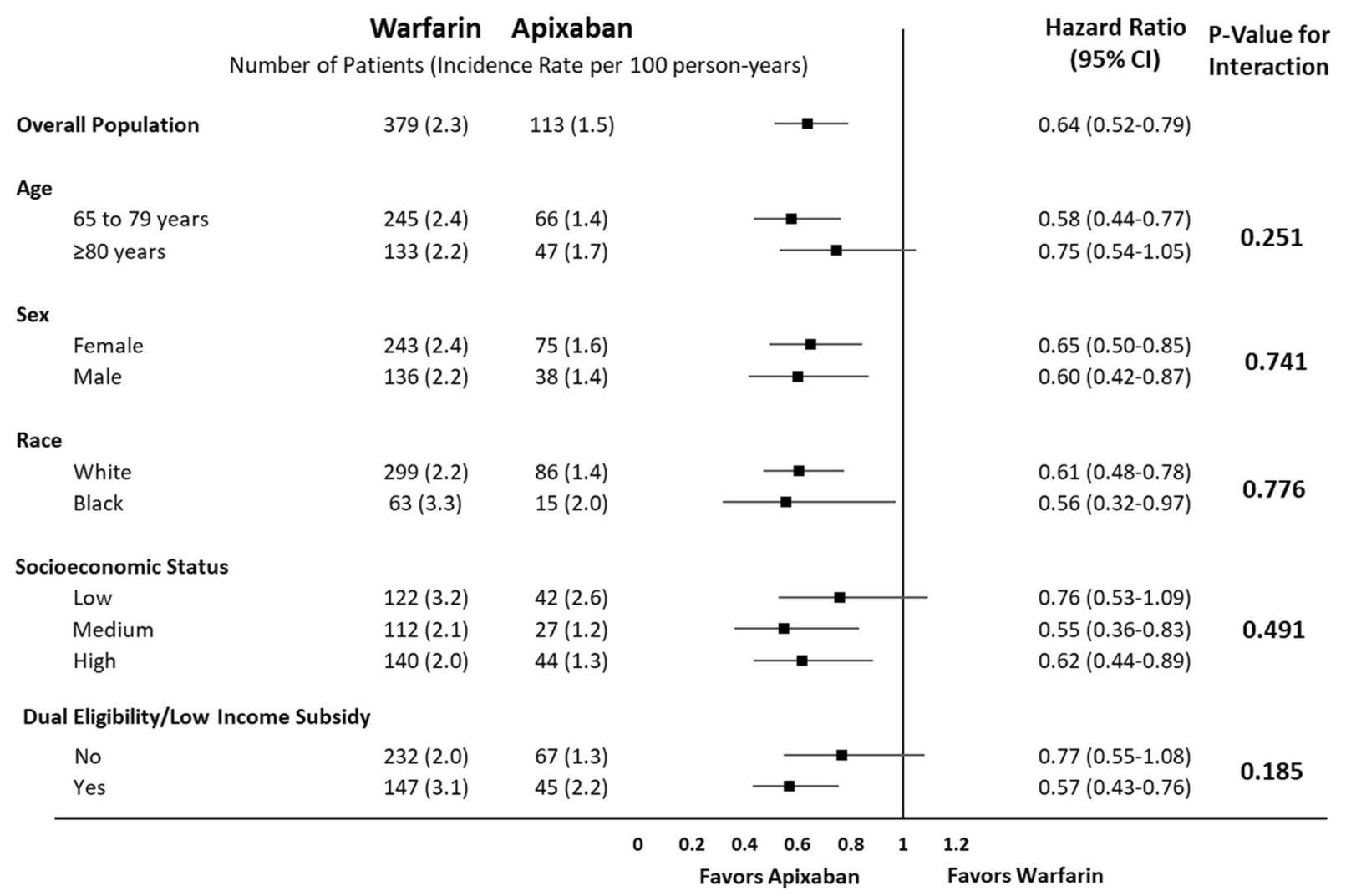

Fig. 2 Risk of recurrent VTE among older patients with VTE stratified by demographic and socioeconomic factors. CI: confidence interval

factors including age, sex, race, SES, and dual eligibility/Part-D LIS. In patients with VTE aged $\geq 65$ years, apixaban was associated with significantly lower risk of recurrent VTE, MB, and CRNMB compared to warfarin. When stratified by the demographic and socioeconomic factors, black patients and patients with lower SES tended to have higher incidence rates of recurrent VTE, MB, and CRNMB than their respective counterparts for both apixaban and warfarin cohorts. Comparisons of apixaban with warfarin on recurrent VTE and MB by the demographic and socioeconomic factors show no significant interaction between the treatment and the following subgroups: age, sex, race, SES, and dual eligibility/Part-D LIS. Two significant interactions were observed for CRNMB, but apixaban patients trended towards a lower risk of CRNMB across all subgroup strata.
Our findings are generally consistent with the age and gender subgroup analysis of the AMPLIFY clinical trial. AMPLIFY demonstrated that apixaban was noninferior to enoxaparin followed by warfarin for the treatment of VTE and was associated with significantly less bleeding among patients with VTE [13]. For AMPLIFY, approximately $58 \%$ of patients were male with an average age of 57 years [13]. In the subgroup analysis of AMPLIFY, there was no significant interaction between treatment and age subgroups (aged $<65$ years, $65-74$ years, and $\geq 75$ years, interaction $p=0.3427$ ) as well as between treatment and gender (interaction $p=0.4514$ ) with regards to recurrent VTE or VTE-related death [13]. There was also no significant interaction between treatment and age (interaction $p=0.8174$ ) or between treatment and gender (interaction $p=0.4168$ ) with regards to $\mathrm{MB}$ [13]. Consistently, our study did not observe significant interactions between the 


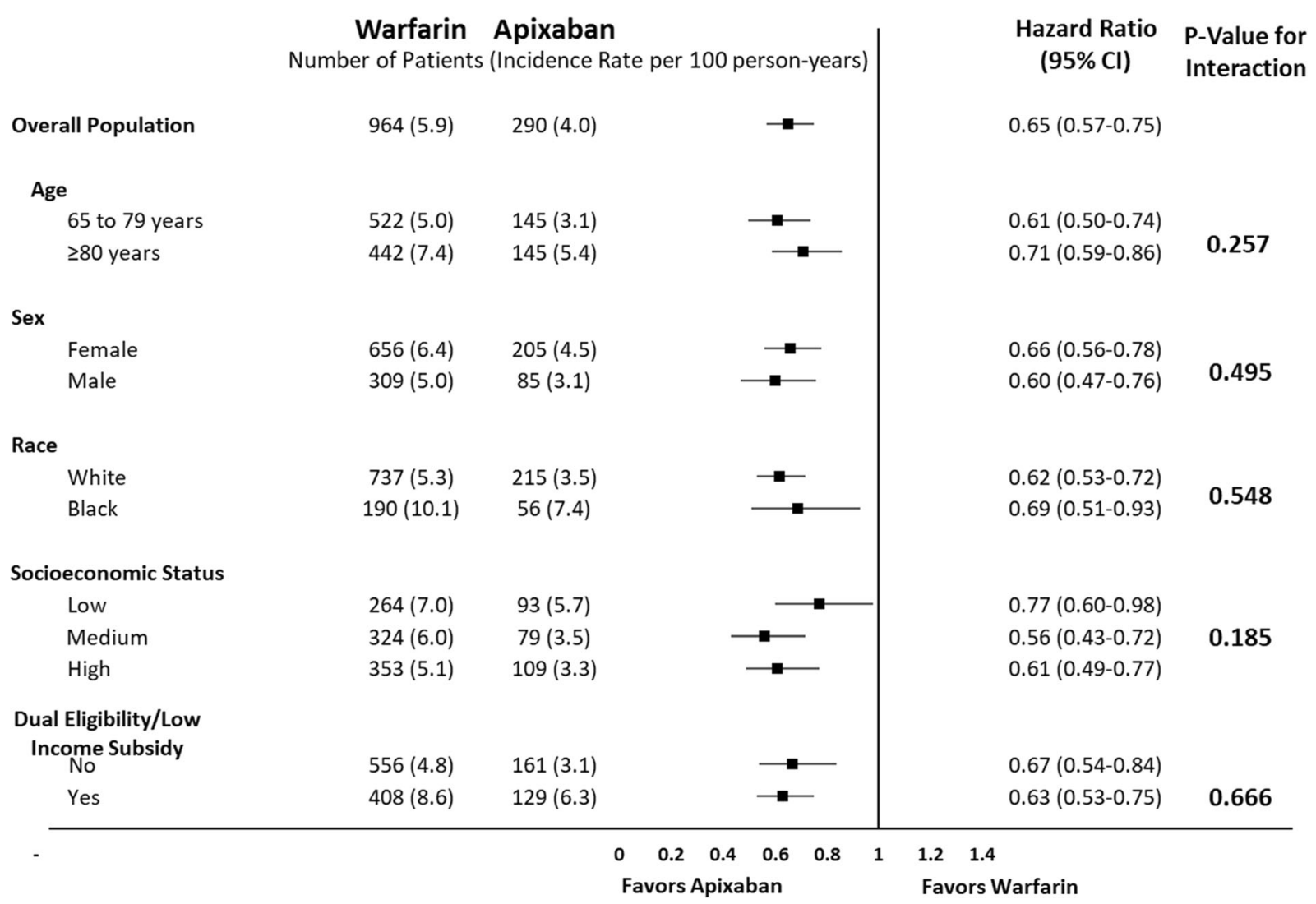

Fig. 3 Risk of major bleeding among older patients with VTE stratified by demographic and socioeconomic factors. CI: confidence interval

treatment and age or gender for recurrent VTE or MB.

Race has been found to be a risk factor for VTE and racial disparity in VTE event rates has been well documented in the literature [25-27]. In general, black patients were found to have a higher incidence and risk of VTE compared to white patients [25-27] possibly due to underlying risk factors for VTE compared to white patients as well as low adherence to treatment [28]. Consistent with this, the current study found that black patients had a higher incidence rate per 100 person-years for recurrent VTE compared to white patients. Additionally, we found a higher incidence rate of $M B$ and CRNMB for black vs white patients. A previously published single-center study also showed that among DVT patients, race (non-white racerelative hazard 1.6; 95\% CI 1.2-2.1) was a risk factor for bleeding during hospitalization [29]. Despite the higher risk of VTE among black patients, a retrospective study found that black patients with VTE were significantly less likely to receive a DOAC treatment compared to white patients (odds ratio [OR] 0.86; 95\% CI $0.77-0.97)$ [12]. In this study of apixaban vs warfarin, we did not observe any significant interaction between treatment and race for recurrent VTE, MB, or CRNMB. The treatment effects of apixaban vs warfarin were consistently observed between black and white patients. Similarly, most of the subgroup analyses of the DOAC pivotal trials in patients with VTE did not find significant differences in treatment outcomes by race [6]. More studies are needed to understand the reasons for the racial disparity in VTE treatments.

Disparities in VTE treatments also exist among patients with different SES. A retrospective analysis of patients with VTE reported that patients with an income of greater than US\$100,000/year were more likely to receive 


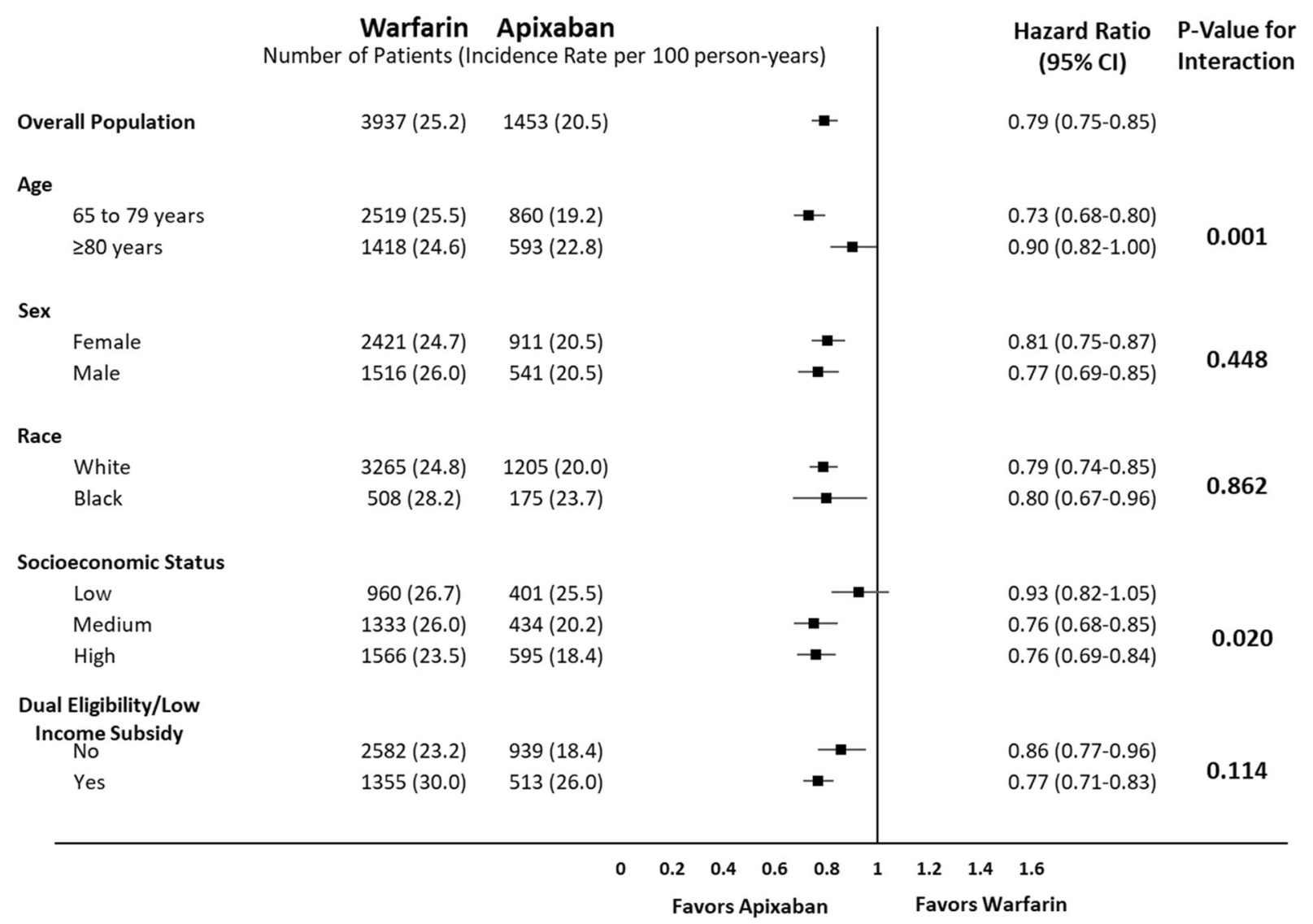

Fig. 4 Risk of clinically relevant non-major bleeding among older patients with VTE stratified by demographic and socioeconomic factors. CI: confidence interval

DOAC therapy compared with patients with an income of less than US $\$ 40,000$ per year (OR 1.50; 95\% CI 1.33-1.69) after controlling for other factors [12]. To address the treatment disparity, it is important to understand whether DOAC treatment effects differ by different SES. In the current study, we compared apixaban with warfarin for recurrent VTE, MB, and CRNMB stratified by SES and by Medicare Medicaid dual eligibility or LIS which is a group of vulnerable patients with low income and complex medical needs. No significant interaction was found between the treatment and SES or between the treatment and dual eligibility/ LIS for recurrent VTE and MB. The treatment effects of apixaban vs warfarin on recurrent VTE and $\mathrm{MB}$ were consistently observed regardless of SES and dual eligibility/LIS. Although a significant interaction was found between the treatment and SES on CRNMB, apixaban patients trended towards a lower risk of CRNMB across all strata of SES. Additional efforts are needed to optimize VTE treatments for patients with low SES.

As with any retrospective claims analysis, the current study has several limitations. First, the definitions of recurrent VTE and MB were based on inpatient claims with primary diagnosis code for VTE and MB, respectively. While the use of inpatient primary diagnosis would ensure that the recurrent VTE and MB events being captured are indeed major acute events, such definitions may be too strict to cause underestimates of the events. Moreover, the definition for CRNMB has not been validated in the literature, although it attempts to align with the 
definition suggested by the International Society on Thrombosis and Haemostasis [19]. Second, the race categories used in the study were based on what have been defined in the Medicare database and a small percentage of patients in the database had unknown race. It is also possible that there could be some misclassification of race in the database. Third, the SES was based on zip codes rather than the income of the patients. However, studies have shown that zip code level classification of SES is a more conservative proxy and is often used [30-32]. Fourth, only association rather than causation could be inferred and hence the results should be interpreted with caution. Fifth, the baseline medications included in this study were mostly limited to those that were likely to increase the risk of bleeding or clots. This list may not be comprehensive, and some other important medications may be missed. Sixth, polypharmacy was not adjusted for in the model and could have an impact on risk of recurrent VTE and bleeding. Lastly, the results of this study cannot be generalized to older patients with other insurances such as Medicare Advantage, Veterans Affairs, or those who are uninsured.

\section{CONCLUSIONS}

In this study of patients with VTE aged $\geq 65$ years, higher incidence rates of recurrent VTE, MB, and CRNMB were observed in black vs white patients and patients with lower vs higher SES. Apixaban had a lower risk of recurrent VTE, MB, and CRNMB compared to warfarin. The treatment effects of apixaban vs warfarin were generally consistent across the demographic and socioeconomic subgroups. More studies are needed to identify optimal VTE management strategies for black patients and patients with lower SES.

\section{ACKNOWLEDGEMENTS}

Funding. Sponsorship for this study, including payment for the rapid service and open access fees, was provided by Pfizer and Bristol Myers Squibb Company.

Authorship. All named authors meet the International Committee of Medical Journal Editors (ICMJE) criteria for authorship for this article, take responsibility for the integrity of the work as a whole, and have given their approval for this version to be published.

Author Contributions. Conceptualization: all authors; Methodology: all authors; Formal analysis and investigation: Janvi Sah and Allison Keshishian; Writing - original draft preparation: Janvi Sah and Allison Keshishian; Writing - review and editing: all authors; Funding acquisition: Theodore Lee, Lisa Rosenblatt, Patrick Hlavacek, Birol Emir, and Xuemei Luo; Resources: Huseyin Yuce; Supervision: Alexander Cohen.

Medical Writing, Editorial, and Other Assistance. Editorial services were provided by Chris Haddlesey of STATinMED Research, a paid consultant to the study sponsors - Pfizer and Bristol Myers Squibb Company - in connection with the manuscript.

Disclosures. Alexander T. Cohen receives consulting fees and research support from various companies including Abbvie, Alexion Pharmaceuticals, Bayer, BMS, Daiichi-Sankyo, Janssen, Johnson \& Johnson and Pfizer. Janvi Sah and Allison Keshishian are employees of STATinMED Research, a paid consultant to the study sponsors in connection with the manuscript. Theodore Lee, Patrick Hlavacek, Birol Emir, and Xuemei Luo are employees of Pfizer, one of the study sponsors. Amol D. Dhamane and Lisa Rosenblatt are employees of Bristol Myers Squibb Company, one of the study sponsors. Huseyin Yuce have no conflicts of interest to disclose.

Compliance with Ethics Guidelines. This retrospective database analysis did not involve the collection, use, or transmittal of individual identifiable data. As such, IRB approval to conduct this study was not required and considered exempt according to 45CFR46.101(b)(4): 
Existing Data \& Specimens - No Identifiers. Both the data set itself and the security of the offices where the data are housed meet the HIPAA requirements of 1996 .

Data Availability. The datasets generated during and/or analyzed during the current study are available from the corresponding author on reasonable request.

Open Access. This article is licensed under a Creative Commons Attribution-NonCommercial 4.0 International License, which permits any non-commercial use, sharing, adaptation, distribution and reproduction in any medium or format, as long as you give appropriate credit to the original author(s) and the source, provide a link to the Creative Commons licence, and indicate if changes were made. The images or other third party material in this article are included in the article's Creative Commons licence, unless indicated otherwise in a credit line to the material. If material is not included in the article's Creative Commons licence and your intended use is not permitted by statutory regulation or exceeds the permitted use, you will need to obtain permission directly from the copyright holder. To view a copy of this licence, visit http://creativecommons.org/licenses/by$\mathrm{nc} / 4.0 /$.

\section{REFERENCES}

1. Galson SK. The Surgeon General's call to action to prevent deep vein thrombosis and pulmonary embolism. https://www.ncbi.nlm.nih.gov/books/ NBK44178/. Accessed 1 Apr 2021.

2. Ho KM, Litton E. Venous thromboembolism prophylaxis in hospitalized elderly patients: time to consider a 'MUST' strategy. J Geriatr Cardiol. 2011;8(2):114-20.

3. Heit JA, O'Fallon WM, Petterson TM, et al. Relative impact of risk factors for deep vein thrombosis and pulmonary embolism: a population-based study. Arch Intern Med. 2002;162(11):1245-8.

4. White RH, Keenan CR. Effects of race and ethnicity on the incidence of venous thromboembolism. Thromb Res. 2009;123(Suppl 4):S111-7.
5. Aujesky D, Long JA, Fine MJ, Ibrahim SA. African American race was associated with an increased risk of complications following venous thromboembolism. J Clin Epidemiol. 2007;60(4):410-6.

6. Gibson CM, Yuet WC. Racial and ethnic differences in response to anticoagulation: a review of the literature. J Pharm Pract. 2019. https://doi.org/10. 1177/0897190019894142.

7. Zakai NA, McClure LA. Racial differences in venous thromboembolism. J Thromb Haemost. 2011;9(10): 1877-82.

8. Engbers MJ, van Hylckama VA, Rosendaal FR. Venous thrombosis in the elderly: incidence, risk factors and risk groups. J Thromb Haemost. 2010;8(10):2105-12.

9. Goldhaber SZ. Race and venous thromboembolism: nature or nurture? Circulation. 2014;129(14): $1463-5$.

10. Ortel TL, Neumann I, Ageno W, et al. American Society of Hematology 2020 guidelines for management of venous thromboembolism: treatment of deep vein thrombosis and pulmonary embolism. Blood Adv. 2020;4(19):4693-738.

11. Kearon C, Akl EA, Orneloas J, et al. Antithrombotic therapy for VTE disease: CHEST guideline and expert panel report. Chest. 2016;149(2):315-52.

12. Nathan AS, Geng Z, Dayoub EJ, et al. Racial, ethnic, and socioeconomic inequities in the prescription of direct oral anticoagulants in patients with venous thromboembolism in the United States. Circ Cardiovasc Qual Outcomes. 2019;12(4):e005600.

13. Agnelli G, Buller HR, Cohen A, et al. Oral apixaban for the treatment of acute venous thromboembolism. N Engl J Med. 2013;369:799-808.

14. Weycker D, Li X, Wygant GD, et al. Effectiveness and safety of apixaban versus warfarin as outpatient treatment of venous thromboembolism in U.S. clinical practice. Thromb Haemost. 2018;118(11): 1951-61.

15. Hlavacek P, Guo JD, Rosenblatt L, et al. Safety, effectiveness, and health care cost comparisons among elderly patients with venous thromboembolism prescribed warfarin or apixaban in the United States Medicare population. Curr Med Res Opin. 2019;35(12):2043-51.

16. Cohen AT, Keshishian A, Lee T, et al. Effectiveness and safety of apixaban, low-molecular-weight heparin, and warfarin among venous thromboembolism patients with active cancer: a U.S. claims data analysis. Thromb Haemost. 2021;121(3): 383-95. 
17. Cohen A, Sah J, Lee T, et al. Effectiveness and safety of apixaban vs. warfarin in venous thromboembolism patients with obesity and morbid obesity. J Clin Med. 2021;10(2):200.

18. Weycker D, Wygant GD, Guo JD, et al. Bleeding and recurrent VTE with apixaban vs warfarin as outpatient treatment: time-course and subgroup analyses. Blood Adv. 2020;4(2):432-9.

19. Kaatz S, Ahmad D, Spyropoulos AC, Schulman S. Definition of clinically relevant non-major bleeding in studies of anticoagulants in atrial fibrillation and venous thromboembolic disease in non-surgical patients: communication from the SSC of the ISTH. J Thromb Haemost. 2015;13(11):2119-26.

20. Birkmeyer NJ, Gu N, Baser O, Morris AM, Birkmeyer JD. Socioeconomic status and surgical mortality in the elderly. Med Care. 2008;46:893-899.

21. Roux AVD, Merkin SS, Arnett D, et al. Neighborhood of residence and incidence of coronary heart disease. N Engl J Med. 2001;345(2):99-106.

22. Austin PC, Stuart EA. Moving towards best practice when using inverse probability of treatment weighting (IPTW) using the propensity score to estimate causal treatment effects in observational studies. Stat Med. 2015;34(28):3661-79.

23. Thoemmes F, Ong AD. A primer on inverse probability of treatment weighting and marginal structural models. Emerg Adulthood. 2016;4(1):40-59.

24. Xu S, Ross C, Raebel MA, Shetterly S, Blanchette C, Smith D. Use of stabilized inverse propensity scores as weights to directly estimate relative risk and its confidence intervals. Value Health. 2010;13(2): 273-7.
25. Dowling NF, Austin H, Dilley A, Whitsett C, Evat $\mathrm{BL}$, Hooper WC. The epidemiology of venous thromboembolism in Caucasians and AfricanAmericans: the GATE Study. J Thromb Haemost. 2003;1(1):80-7.

26. Schneider D, Lilienfeld DE, Im W. The epidemiology of pulmonary embolism: racial contrasts in incidence and in-hospital case fatality. J Natl Med Assoc. 2006;98(12):1967-72.

27. Keenan CR, White RH. The effects of race/ethnicity and sex on the risk of venous thromboembolism. Curr Opin Pulm Med. 2007;13(5):377-83.

28. Arnsten JH, Gelfand JM, Singer DE. Determinants of compliance with anticoagulation: a case-control study. Am J Med. 1997;103(1):11-7.

29. White RH, Beyth RJ, Zhou H, Romano PS. Major bleeding after hospitalization for deep-venous thrombosis. Am J Med. 1999;107(5):414-24.

30. Thomas A, Eberly L, Smith G, et al. ZIP-code-based versus tract-based income measures as long-term risk-adjusted mortality predictors. Am J Epidemiol. 2006;164:586-90.

31. Krieger N, Chen J, Waterman P, et al. Geocoding and monitoring of US socioeconomic inequalities in mortality and cancer incidence: does choice of area-based measure and geographic level matter? Am J Epidemiol. 2002;156:471-82.

32. Krieger N, Chen J, Waterman P, et al. Race/ethnicity, gender, and monitoring socioeconomic gradients in health: a comparison of area based socioeconomic measures. Am J Public Health. 2003;93:1655-71. 